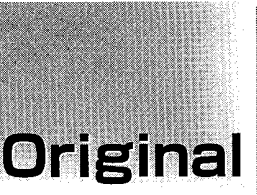

Received July 12, 2005

Revision accepted

Oct. 21, 2005

Code No. 111

\section{Analysis of Medical Accidents Using the "Why Why Why Analysis" (Questions): Comparison with Conventional Analytical Techniques}

\section{HITOSHI TSUCHIYA, HIROMASA KUROSAKI ${ }^{1}$, SOICHI HIRAMOTO, MISAYO SEKI, KEIZABURO MORIUCHI, SATORU KAWAUCHI, and ISAO KURITA}

Department of Radiology, Toranomon Hospital

1 ) Department of Radiology, Funabashi Municipal Center

\section{Introduction}

Although there are numerous reports on medical risk management, ${ }^{1-3)}$ the majority of these deal with understanding accident events and are unable to make active use of accident information as a result of failing to extract factors common to the occurrence of errors. The reason for this is a lack of methodology for systematically implementing countermeasures to enable anyone to prevent accidents. For this reason, the development of accident countermeasures was considered to require experienced personnel having both knowledge and experience relating to the organizational background and work. In general, the establishment of a system for preventing errors that occurred in the past has conventionally been the basic policy for preventing accidents. However, this conventional approach prevents all persons from developing a strategy of countermeasures.

Although the "Why Why Why Analysis Method"4,5) used in industry is considered to be a minor technique, it is currently used by numerous private corporations. The main factor that propelled this technique to fame was its use by Toyota Motor Corporation in its "The 5 Whys." Although this technique was brought to center stage with its use by Taiichi Oono in his research and development on improving operations, it was actually developed and used in Japanese industry as the "Why Why Why Questions." The Department of Radiology at Toranomon Hospital developed a countermeasure strategy by performing "Why Why Why Analysis" on medical accidents and problems that actually occurred at the hospital. A comparative study was conducted with other methods of analysis, including case studies obtained from experiences at the hospital, the results of which are reported here.

\section{Materials and Methods}

A study of proposed accident countermeasures was conducted using "Why Why Why Analysis" by the ac-

\title{
Summary
}

Techniques such as 4M-4E matrices and the SHEL model have been proposed in recent years as tools for analyzing medical accidents and developing countermeasures. There have been hardly any reports, however, describing their use for the development of risk strategies in the medical setting. After using the SHEL model, Toranomon Hospital is currently using "Why Why Why Analysis" and has had several successes as a result of its use. With this in mind, a comparative study was conducted between "Why Why Why Analysis" and several previously reported accident countermeasure tools, root cause analysis (RCA), used at Veterans Hospitals in the US, and quality control (QC), used in the industrial sector. As a result, "Why Why Why Analysis" as applied in radiology work was determined to be easy to deploy even for beginners as compared with the other tools, able to accommodate complaints as well as accidents, and useful on the basis of having both practical and expandable functions for improving radiology work.

Key words: Risk management, Why Why Why, Quality control, SHEL model, 4M-4E matrices 
cident prevention committee of the Department of Radiology of Toranomon Hospital. During that analysis, a work flowchart was prepared and examined for the purpose of understanding the overall work process.

On the basis of that experience, a comparative study was conducted on "Why Why Why Analysis" with the SHEL model, ${ }^{6)} 4 \mathrm{M}-4 \mathrm{E}$ matrices, ${ }^{7)}$ root cause analysis (RCA) ${ }^{8)}$ and quality control. ${ }^{9)}$

\section{Results}

\section{2-1 Case Study}

[Description]

Tests were performed in the X-ray room without noticing that the date setting on the digital radiography unit was incorrect, resulting in films being submitted with different date displays.

[Analysis]

Compound factors were not found, since the problem was extremely simple. The cause was suspected of being human error resulting from preconceptions of technicians, physicians, and the manufacturer. The date is displayed at two locations on the film as the test date and image output date. Although the test date and image output date are typically the same, the dates may differ if filming is performed on a later date. These detailed data are believed to have indirectly caused the delay in discovering the incorrect dates. Specific measures were examined for "decreased attentiveness," "poorly distinguishable dates," and "preconceptions of being correct," focusing primarily on time analysis (Fig.).

[Countermeasures]

(1) Remaking of the start-of-work inspection chart (additional confirmation of date)

A revised edition of the inspection chart had not been made since it was initially developed two years previously. The reason for not reviewing the chart was a lack of any new equipment changes. Checking the date first thing in the morning by all personnel, not only by the testing supervisor, will lead to a decrease the number of fundamental mistakes (double check).

(2) Allocation of a single location for displaying the date on films

The date is currently displayed at one or two locations, depending on the type of test. Although there are reasons for each, the radiology department will consider moving in the direction of unifying the display of date at a single location.
(3) Confirmation of clock on PC tower

The battery will be replaced once a year at the time of maintenance and inspection. The battery is considered to be operable for roughly five years. It was decided to replace the battery once a year in consideration of the potential of the battery itself being defective and its low cost.

\section{2-2 Comparison of "Why Why Why Analysis" and SHEL Model Analysis}

SHEL model analysis is a technique used for aircraft accidents. It can be said to be a kind of accident analysis that is performed to maintain normal operations. There are four factors that have an effect on the persons involved in the form of humans maintaining optimum status. Accommodations are considered according to the persons involved and the four factors so that the abilities and limitations of the central person change according to the situation. The central L (oneself) considers accommodations according to the four factors. This has recently come to be expressed as the p-m SHEL model as a result of adding the factors of management $(\mathrm{m})$ and patient (p). It is prerequisite of this model to be able to provide specific preventive measures, including irregular events, as suitable conditions as a model for preventing accidents in the medical setting. Although this technique is advantageous in terms of being simple and facilitating easy analysis, it has limitations in terms of extracting a large number of factors, and has difficulty identifying areas of improvement in the case of compound accidents.

\section{2-3 Comparison of "Why Why Why Analysis" and 4M-4E Matrices}

$4 \mathrm{M}-4 \mathrm{E}$ matrices analysis is a tool that was developed for the purpose of analyzing and investigating accidents at the US National Aeronautics and Space Administration(NASA). By classifying accident factors determined through investigations into four factors and constructing countermeasure proposals required for carrying out a mission in four fields, it is possible to identify what types of problems must be dealt with for the accident factors from a broad perspective. The advantages of this classification method is that it facilitates identification of problems encountered by specific departments within an organization and what needs to be done to deal with these problems. According to a report comparing 4M-4E matrices and the SHEL model, 4M- 


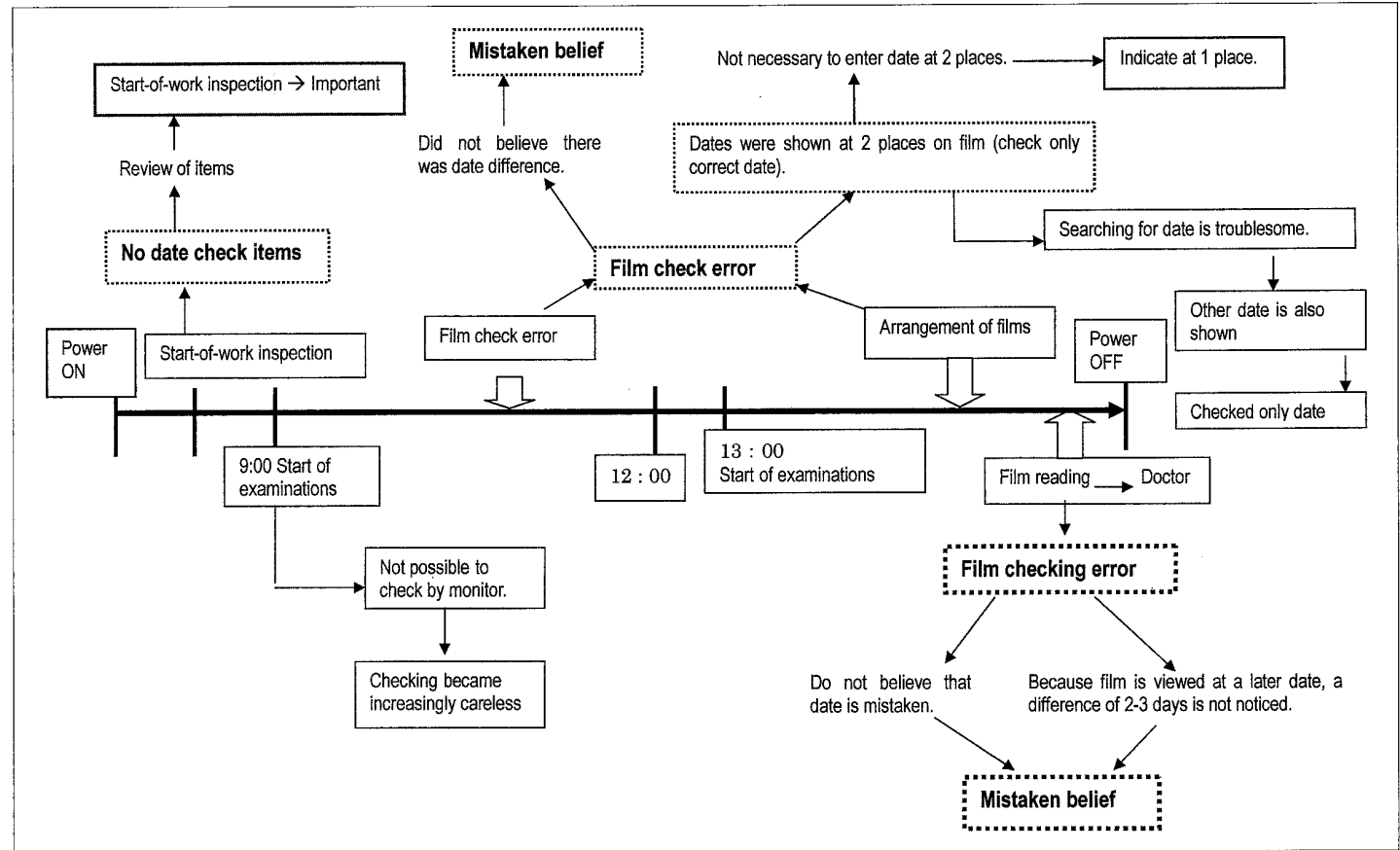

Fig. Date error in digital radiography unit (partial excerpt of analysis).

4E matrices is indicated has facilitating extraction of accident factors due to the larger number of parameters, thus making it useful for organizational improvement. However, in the medical setting, it is not necessary to identify areas of improvement and analyze all factors. There is no guarantee that all 16 parameters can be classified for irregular factors, and since it is impossible to reduce human error to zero in the medical setting, a technique that enables an error-tolerant approach is considered to be more useful.

\section{2-4 Comparison of "Why Why Why Analysis" and RCA}

RCA is a technique used to analyze the root cause of a problem. According to a report from Nerima General Hospital, ${ }^{8)}$ where this technique is currently being used in Japan, the technique was originally developed at the veterans' affairs hospitals. Although the health care organization and system in the US and Japan cannot be said to be the same, this technique may be the optimum tool for use as a medical accident strategy. RCA is the same with respect to seeking latent system problems and human factor problems by going back from an accident after a problem or accident has occurred. Intensive discussions are held with members from other organizations to identify the cause and clarify latent problems followed by developing policies for improvement and carrying out exhaustive follow-up investigations. The major differ- ence between this technique and "Why Why Why Analysis" is that it allows proposed countermeasures to be developed immediately, since the objective of "Why Why Why Analysis" is not to lead to the identification of the root cause. However, since "Why Why Why Analysis" does not emphasize determination of the root cause, emphasis is placed on a strategy for countermeasures. Although the consideration of countermeasures based on the root cause is the normal approach, there is not time for this in the medical setting. There is no choice but to develop a strategy that places priority on preventing recurrences in order to prevent accidents that have the potential for recurring on the following day. Work must proceed normally even if the root cause is unknown. The allocation of more than 50 hours to form a team and conduct improvement follow-up investigations is virtually impossible in the health care environment of Japan. However, this does not mean that this technique is not practical for health care in Japan. Even if RCA does not function effectively in Japan's health care environment, which currently lacks the foundation for a culture of safety owing to the absence of a tradeoff between time and the construction of effective strategies and organizations and new service systems, when the foundation for a culture of safety is realized at some point in the future, RCA is expected to yield significant effects together with shortened time and the establishment of a safety management sector. 


\section{2-5 Comparison of "Why Why Why Analysis" and QC}

The concept of QC was introduced to Japanese industry by Dr. W.E.Demming about 50 years ago, and has continued to evolve in the form of QC techniques (QA circles ${ }^{10)}$ employed by various private corporations. However, there is a considerable difference between medicine and industry, since corporate society has already completed a system for dealing with problems involving quality. Since the organizational structure of health care differs greatly from that of the industrial world, progress comes at a much slower pace. Even though a hospital director may be able to state that his hospital is deploying QC, if only QC personnel have an understanding of QC, its effects are limited, and it is believed that quite some time will be required for all staff members to understand the significance of QC. Within the QC technique, a fishbone chart and a relationship chart are used as typical tools for accident countermeasures (7 QC tools). Both have the advantage of facilitating free expression of opinions in the manner of brainstorming. In the case of the fishbone chart, instead of selecting countermeasures after having summarized all factors following the completion of a factor chart, the cause is specified and countermeasures are developed from the unidirectional opinions of the analyst (previous experience contribution rate). However, there are likely to be difficulties in ending the process at this point. In addition, although relationship charts are suitable for systematically focusing on the cause from an overall image (determination of the root cause), it is unlikely that this will lead to the prevention of recurrence.

The results of the comparisons described above are presented in a comparison chart (Table).

\section{Discussion}

The following conclusions can be drawn based on the findings of this case study. The SHEL model begins with "who" (person involved) was unable to find the incorrect date setting. The problem of who was unable to find the incorrect setting can only be extracted from problems relating to hardware, problems relating to software, the person involved, and the relationship with his or her surroundings. In the case of the 4M-4E matrices, the problem is only extracted from problems between two points (intersection of the vertical axis and horizontal axis), thereby making it difficult to extract the problem. In reality, compound causes are suggested to be behind the person involved in overlooking the incorrect date setting such as (1) believing that the date on the computer would probably never be incorrect (physicians, technicians), (2) a defect in the computer system (failing to double-check the dates on the films), and (3) deficiencies in the work manual. It is difficult to analyze these types of compound errors on a matrix. However, in the case of analyzing single errors, it is advantageous to not focus on the system configuration by simplifying the cause of an accident since this makes it easier to extract countermeasure proposals. With respect to QC techniques and QA circles, the determination of the cause is left only to the result, and is avoided by changing the inspection manual used at the start of work as well as maintenance and inspection. However, these types of accident strategies alone are unlikely to be able to eliminate the psychological factors inherent in the technician. One of the major differences between health care and industry is that health care is not systematic. In addition, in the case of RCA, a study is conducted in which detailed data are gathered from the technician who overlooked the incorrect date setting and each datum is analyzed to find the root causes and devise countermeasures for all of those causes. This, however, requires an excessive amount of time. Although it cannot be denied that the deployment of accident countermeasures takes a considerable amount of time, health care operations cannot wait for these countermeasures to be deployed. This technique can be said to be useful for analyzing compounded (overlapping) causes by focusing on the work system and process.

The accident prevention committee of the Department of Radiology of Toranomon Hospital has not used "Why Why Why Analysis" since the time of its establishment. At the time of our establishment, we did not have any analytical tools, but rather employed a method involving the gathering of opinions in the form of incident reports. This approach was only effective in identifying single causes, and only allowed errors to be avoided by providing rules or equipment. In reality, however, such errors are few in number, and instead consist predominantly of compound human errors. ${ }^{11}$ ) Thus, this approach was ineffective in the development of countermeasures, and there were repeated recurrences. In other words, the majority of errors are not single errors, but rather errors that manifest as a result of compound causes involving relationships between people (health care personnel and patients, health care personnel and other health care personnel) and the environment, 


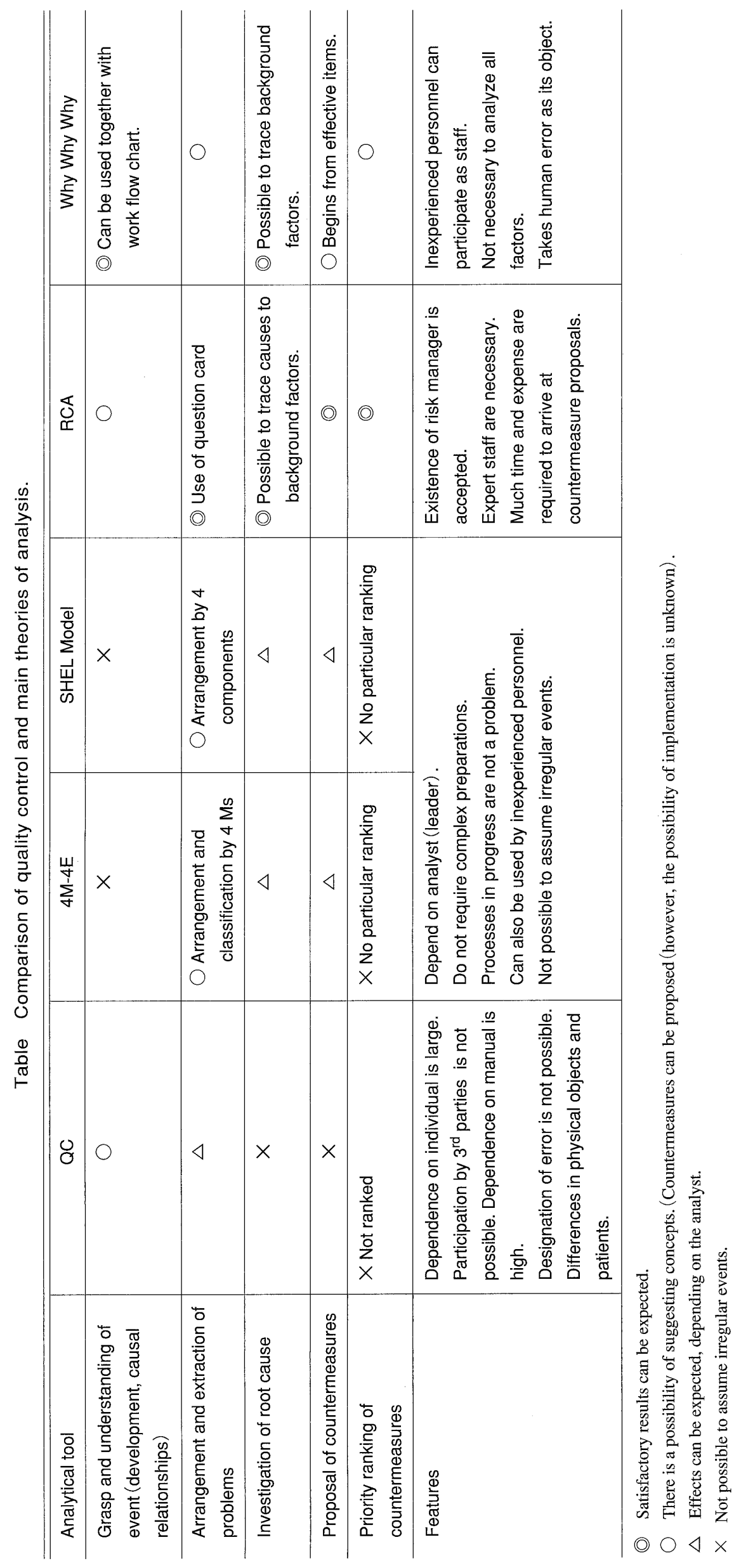

第 61 卷 第 12 号 
between people, between the work process and the environment, and so forth.

Subsequently, we proposed accident countermeasures using several techniques (4M-4E matrices, SHEL model) until we arrived at the "Why Why Why Analysis." However, despite having presented numerous proposed countermeasures, hardly any of them were used. More precisely, although some of them may have been used, they were unable to generate any significant results. It was not even possible to evaluate the effects of accident countermeasures. More specifically, we were only able to generate answers that would have been recognized by anyone without having to hold a special meeting of the committee, and the accident countermeasures that were proposed were largely dependent on individual temperament. We therefore searched for a technique of analysis that matched our needs. During the course of that search, it was necessary for committee members to be able to develop accident countermeasure strategies without having the experience of analyzing case studies, to ensure efficacy and reliability even in the absence of a foundation for a culture of safety in hospitals, and to derive a management technology with a high possibility of being implemented while also retaining flexibility based on the rapid development of a foundation for a culture of safety by health care institutions and the difficulty in investing funds. As a tool for satisfying these essential requirements, the question of "Why did something occur?" enabled us to proceed without having completely to explain the reason for a pattern of questions. At present, this approach has spread not only throughout Japan, but throughout many other countries as well, and is referred to as "Why Why Why Analysis" in the US. The greatest advantage of this technique of analysis is that allows the question of "why" to be asked repeatedly until measures for preventing recurrence have been taken. This is because, instead of focusing on new technologies and new tasks, it simply follows previous theories and principles.

At the time the committee of the Department of Radiology first adopted "Why Why Why Analysis," although it was useful for unitary cause analysis (accident analysis), it was thought to be unsuitable for time and process analysis. However, as a result of producing a "Work Flow Chart," it became possible to perceive accidents in the form of compound errors instead of single errors, thereby making it possible to present accommodating measures as an organization. In addition, veri- fying accidents by members of the committee alone typically resulted in limitations on examining unseen aspects of the work process. Despite this, it was difficult for members from other departments to understand the workflow, and it is normally impossible to determine the overall work process. Since it is normally the objective to seek out faults in work, by introducing an event relationship chart, it became possible to extract compounded accidents by changing the perspective.

There were many opinions recognizing the effectiveness of this technique among members of the accident prevention committee as a result of the technique's introduction. In the past, owing to the small number of experienced personnel in the departments of radiotherapy and nuclear medicine, accident strategies had always been postponed, and it was difficult to develop strategies for preventing recurrence. Risk countermeasures made it difficult to understand the work system and focused primarily on monitoring, while leaving risk countermeasures to the discretion of individuals. In response to this situation, an awareness grew that involved approaching the problem head on. In addition, health care personnel other than committee members suggested the potential for small errors leading to serious accidents, resulting in the establishment of an atmosphere of safety management.

Accident prevention strategies do not begin and end with a determination of the root cause, but rather are believed to require a discussion of proposed countermeasures while searching for the root cause. This is because, if background factors are able to be analyzed at this time, it becomes possible to develop proposals for countermeasures for preventing errors, and even if a countermeasure has been proposed on the basis of cause analysis, since it cannot be easily verified, repeatedly asking "Will a new accident be induced?" and "If not, why not?" on the basis of new countermeasures makes it possible to develop countermeasures in the true sense. Although the process is over if countermeasures are proposed based on an error-proof approach or if the reason for an accident not occurring is determined, in the case of the likelihood of a new error occurring, repeatedly asking why that error occurs is believed to allow the development of countermeasures that prevent its recurrence.

\section{Conclusion}

"Why Why Why Analysis" enables the development of a risk strategy even if it does not allow adequate root cause analysis or background factor analysis in the de- 
velopment of accident countermeasures. Although effective accident prevention strategies are not considered to be proportional to the amount of time spent on brainstorming, in Japan's health care environment, which lacks a risk quality control sector, it is at least necessary to implement a strategy even if that strategy might be inadequate. Even though "Why Why Why Analysis" may be inadequate in terms of a countermeasures strategy, it can be implemented immediately while also having the major advantage of allowing corrected strategies to be introduced as necessary. The impetus behind the implementation of the "Why Why Why Analysis" reported here was the occurrence of overexposure accidents at this hospital. This case study was then used to make modifications in "Why Why Why Analysis." This tool can be used not only in the radiology department, but also in the departments of nuclear medicine and radiotherapy as well as accommodating complaints from patients, and was suggested to be a practical tool having expandable functions.

\section{Acknowledgments}

We gratefully acknowledge the help of Masaharu Kitamura, Ph.D., Toshio Wakabayashi, Ph.D., and Makoto Takahashi, Ph.D., Management of Science \& Technology Department (MOST), Graduate School of Engineering, Tohoku University.

\section{References}

1) Hayabuchi N, Endoh M, Hirokawa Y, et al.: Risk Management in Radiation Therapy - From a Report by the Excessive Radiation Survey Team of the Hirosaki National Hospital. Japanese Society for Therapeutic Radiology and Oncology, 16 (3), 133-141, (2004).

2) Kumagaya T: Research on Risk Management in Radiation Therapy. Jpn J Radiol Technol, in Japanese, 46, 1-3, (1999).

3) Amauchi H: Risk Management in the Radiology Department (1) - (3). Jpn J Radiol Technol, in Japanese, 48, 6-8, (2001).

4) Ogura N: Effective Techniques for Utilization of Why Why Why Analysis. Japan Institute of Plant Maintenance. in Japanese, (2003).

5) Takayanagi $M$, Mihashi $H$, and Orihata $J$ : Ultimate Work Techniques Learned from Toyota Employees 10, Nikkei Business Associe, in Japanese, 24-53, November 16, (2004).

6) Japanese Nursing Association, ed., Medical Accident Preven- tion on an Organizational Basis, Japanese Nursing Association Publishing, (2000).

7) Ministry of Health, Labor and Welfare, Health Policy Bureau, General Affairs Division, Case of Misrecognition of a Patient at Yokohama City University, (1999).

8) Yanagigawa T: Accident Report Analysis Improvement Systems and RCA. J Natl Inst Public Health, in Japanese, 51 (4), (2002).

9) Maruno H: Towards the Establishment of a QA Committee in the Department of Radiology of Toranomon Hospital. Annual Meeting of the Japanese Society for Therapeutic Radiology and Oncology, in Japanese, 256, (1999).

10) Uchida M: Basic of Quality Control Nihonkeizaishinbunsya, in Japanese, (2001).

11) Kohn LT: To Err is Human, Medical Journalists Association of Japan trans. Nippon-Hyoron-Sha Co., Ltd., (1999). 